\title{
One-loop divergences of the Einstein-Yang-Mills system
}

\author{
S. Deser,${ }^{*}$ Hung-Sheng Tsao ${ }^{\dagger}$ and P. van Nieuwenhuizen ${ }^{\ddagger}$ \\ Department of Physics, Brandeis University, Waltham, Massachusetts 02154
}

(Received 1 July 1974)

\begin{abstract}
The one-loop ultraviolet divergences of the quantized coupled Einstein-Yang-Mills fields are evaluated. They are precisely of the same nonrenormalizable form as for a corresponding set of free Maxwell fields together with the usual flat-space Yang-Mills divergences. The two free Yang-Mills field parameters, namely the group dimension and the self-coupling constant, cannot be chosen to make the one-loop divergences renormalizable in the usual sense.
\end{abstract}

\section{INTRODUCTION}

The recent successes of covariant quantization methods in dealing with non-Abelian vector fields have inspired their application to the theory of the gauge meson of spin 2, Einstein's general relativity. The fundamental difference between these two systems lies in the dimensional character of the gravitational coupling constant $\kappa$, with accompanying momentum dependence of graviton vertices. The latter behave as $p^{2}$, so that even at the oneloop level, every "ring" diagram with arbitrarily many external graviton lines remains divergent, in contrast to the vector case. However, when using the so-called background field method, ${ }^{1-3}$ the fact that the corresponding a mplitudes must be invariant with respect to the gauge transformations on the external lines means that all divergences at the one-loop level can be determined from invariance considerations, plus calculation of a few simple one-loop diagrams. In this way, 't Hooft and Veltman ${ }^{2}$ were able to obtain a lemma which enables one to calculate the sum of all one-loop ultraviolet divergences for a wide class of theories. These include general relativity in interaction with a variety of matter systems, in which both graviton and matter are quantized. For source-free general relativity, the one-loop counterterms vanish for the $S$ matrix ${ }^{2}$ but this result is due to a peculiar identity. The coupled graviton-scalar field system is one-loop nonrenormalizable. ${ }^{2}$ The same method, when applied to the coupled photon-graviton system, again yields nonrenormalizable counterterms, even though a great many possible divergent terms are in fact absent. ${ }^{3}$ It also turns out that fermiongraviton coupling is nonrenormalizable,$^{3}$ though rather different calculations are required in this case, to which the lemma is not applicable.

Hence, we now know that the quantized interacting graviton-spin-0, - spin- $\frac{1}{2}$ and - spin-1 systems require counterterms which cannot be absorbed by rescaling of the bare Lagrangian parameters. ${ }^{4}$
It is a natural question whether matter systems with appropriate self-interactions give better behavior than the free fields treated so far. In this paper, we deal with the obvious choice: YangMills fields (of general semisimple gauge groups), which themselves have a high degree of gauge symmetry. There are now available two new dimensionless parameters, the dimension of the gauge group and the self-coupling constant, whose values we are free to adjust in attempting to cancel the divergences. In Sec. II, we quantize the Einstein-Yang-Mills system, introduce the necessary Faddeev-Popov ghosts, and obtain the effective one-loop action, from which we perform the calculation leading to the final counterterms. Section III is devoted to a qualitative discussion of the result, based on the already known Maxwell case. ${ }^{3}$ In Sec. IV some conclusions and speculations are given.

\section{QUANTIZATION AND COUNTERTERMS}

The Lagrangian for the Einstein-Yang-Mills system is

$$
\begin{aligned}
& \overline{\mathfrak{L}}=\mathcal{L}(\bar{g}, \bar{F})=-(-\bar{g})^{1 / 2}\left[\kappa^{-2} R(\bar{g})+\frac{1}{4} \bar{F}^{2}\right], \\
& \bar{F}_{\mu \nu}^{a} \equiv \partial_{\mu} \bar{V}_{\nu}^{a}-\partial_{\nu} \bar{V}_{\mu}^{a}+f C^{a b c} \bar{V}_{\mu}^{b} \bar{V}_{\nu}^{c}, \quad \bar{F}^{2} \equiv \bar{F}_{\mu \nu}^{a} \bar{F}^{a \mu \nu} .
\end{aligned}
$$

Here, $C^{a b c}$ are the structure constants, $f$ is the self-coupling constant, and other conventions are the same as in Ref. 3.

Following the standard procedure of the background-field method we write

$$
\bar{g}_{\mu \nu}=g_{\mu \nu}+\kappa h_{\mu \nu}, \quad \bar{V}_{\mu}^{a}=V_{\mu}^{a}+A_{\mu}^{a},
$$

where $(g, V)$ are background fields and $(h, A)$ quantum fields. We now expand the Lagrangian $\overline{\mathcal{L}}$ in quantum fields about the background fields. From 
the linear term we get the equations of motion for the classical background fields

$$
G_{\mu \nu}=-\frac{1}{2} \kappa^{2} T_{\mu \nu}, \quad D_{\alpha}^{a b} F_{b}^{\alpha \beta}=0,
$$

where $T_{\mu \nu} \equiv F_{\mu \nu}^{2}-\frac{1}{4} g_{\mu \nu} F^{2}, G_{\mu \nu} \equiv R_{\mu \nu}-\frac{1}{2} g_{\mu \nu} R$, and $D_{\alpha}^{a b} \equiv d_{\alpha} \delta^{a b}-f C^{a b c} V_{\alpha}^{c}$ with $d_{\alpha}$ the gravitationally covariant derivative $(D h=d h)$. The quadratic part, $\mathfrak{L}_{2}$, of $\overline{\mathfrak{L}}$ has the form

$$
\begin{aligned}
\mathscr{L}_{2}=(-g)^{1 / 2} & {\left[-\frac{1}{2}\left(D_{\nu} h_{\alpha \beta}\right) P^{\alpha \beta \rho \sigma}\left(D^{\nu} h_{\rho \sigma}\right)+\frac{1}{2}\left(h_{\mu}-\frac{1}{2} D_{\mu} h\right)^{2}\right.} \\
& \left.-\frac{1}{2}\left(D_{\nu} A_{\mu}^{a}\right)^{2}+\frac{1}{2}\left(D^{\mu} A_{\mu}^{a}\right)^{2}+\frac{1}{2} h_{\alpha \beta}\left(X_{g}+X_{f}\right)^{\alpha \beta \rho \sigma} h_{\rho \sigma}+\frac{1}{2} h_{\alpha \beta} Q_{a}{ }^{\alpha \beta \rho \sigma}\left(D_{\rho} A_{\sigma}^{a}-D_{\sigma} A_{\rho}^{a}\right)+\frac{1}{2} A_{\mu}^{a} Z_{a b}^{\mu \nu} A_{\nu}^{b}\right],
\end{aligned}
$$

where

$$
\begin{aligned}
& P^{\alpha \beta \rho \sigma}=\frac{1}{4}\left(g^{\alpha \rho} g^{\beta \sigma}+g^{\alpha \sigma} g^{\beta \rho}-g^{\alpha \beta} g^{\rho \sigma}\right), \\
& X_{g}^{\alpha \beta \rho \sigma}=P^{\alpha \beta \rho \sigma} R-g^{\alpha \rho} R^{\beta \sigma}+g^{\alpha \beta} R^{\rho \sigma}+R^{\alpha \rho \beta \sigma}, \\
& X_{f}^{\alpha \beta \rho \sigma}=\kappa^{2}\left(P^{\alpha \beta \rho \sigma} \frac{1}{4} F^{2}-\frac{1}{2} F_{a}^{\alpha \rho} F_{a}^{\beta \sigma}-g^{\alpha \rho} F_{2}^{\beta \sigma}+\frac{1}{2} g^{\alpha \beta} F_{2}^{\rho \sigma}\right), \\
& Q_{a}^{\alpha \beta \rho \sigma}=\kappa\left(2 g^{\alpha \rho} F_{a}^{\beta \sigma}-\frac{1}{2} g^{\alpha \beta} F_{a}^{\rho \sigma}\right), \\
& Z_{a b}^{\mu \nu}=-2 f C_{a b}^{c} F_{c}^{\mu \nu}+R^{\mu \nu} \delta_{a b}, \\
& \text { with } \\
& \quad F_{2}^{\rho \sigma} \equiv F_{a}^{j \lambda} F_{\lambda}^{a \sigma} .
\end{aligned}
$$

The above symbols are to be symmetrized and antisymmetrized in accordance with the symmetries of their coefficients in (2.4). The action is invariant under the infinitesimal gauge transformations

$$
\begin{aligned}
h_{\mu \nu}^{\prime}(x)= & h_{\mu \nu}(x)+\left(g_{\mu \lambda} D_{\nu}+g_{\nu \lambda} D_{\mu}\right) \eta^{\lambda} \\
& +\kappa\left[\left(h_{\mu \lambda} D_{\nu}+h_{\nu \lambda} D_{\mu}\right) \eta^{\lambda}+\eta^{\lambda} D_{\lambda} h_{\mu \nu}\right], \\
A_{\mu}^{a}{ }^{\prime}(x)= & A_{\mu}^{a}(x)+\left(V_{\lambda}^{a} D_{\mu} \eta^{\lambda}+\eta^{\lambda} D_{\lambda} V_{\mu}^{a}\right)+D_{\mu} \Lambda^{a} \\
& +A_{\lambda}^{a} D_{\mu} \eta^{\lambda}+\eta^{\lambda} D_{\lambda} A_{\mu}^{a} .
\end{aligned}
$$

As suggested by the form of $\mathscr{L}_{2}$ of Eq. (2.4), we choose the gauge-breaking terms to be

$\mathcal{L}_{B}=-\frac{1}{2}(-g)^{1 / 2}\left(h_{\mu}-D_{\mu} h\right)^{2}-\frac{1}{2}(-g)^{1 / 2}\left(D_{\mu} A^{a \mu}\right)^{2}$.

As in the Maxwell case, the ghost Lagrangian required by $\mathscr{L}_{B}$ can be put in the form

$$
\begin{aligned}
\mathscr{L}_{g}= & (-g)^{1 / 2} \psi^{* \mu}\left(g_{\mu \nu} D^{\lambda} D_{\lambda}-R_{\mu \nu}\right) \psi^{\nu} \\
& +(-g)^{1 / 2} \chi^{* a}\left[-D^{\mu}\left(F_{\mu \nu}^{a} \psi^{\nu}\right)+D^{\mu} D_{\mu} \chi^{a}\right] .
\end{aligned}
$$

In order to apply the lemma of ' $t$ Hooft and Veltman, we must double fields $(h, A)$ and make them complex and redefine $h_{\alpha \beta}^{*} P^{\alpha \beta \rho \sigma} \rightarrow h_{\rho \sigma}^{*}$ and $A_{\alpha}^{* a} g^{\alpha \beta}$ $\rightarrow A_{\beta}^{a}$ to arrive at the standard form

$$
\mathcal{L}=(-g)^{1 / 2}\left[\phi_{i}^{*} \partial_{\nu} \partial^{\nu} \phi_{i}+2 \phi_{i}^{*} N^{\mu, i j} \partial_{\mu} \phi_{j}+\phi_{i}^{*} M^{i j} \phi_{j}\right],
$$

with $\phi_{i}=(h, A, \psi, \chi)$ representing all fields, namely $10 h_{\mu \nu}, 4 r A_{\mu}^{a}, 4 \psi_{\mu}$ and $r \chi^{a}$ where $r$ is the gauge group's dimension. The $N^{\mu}, M$ are matrices in the ( $h, A, \psi, \chi)$ space; they decompose into submatrices in $(h, A)$ and $(\psi, \chi)$. The values of $N^{\mu}$ and $M$ are read off from the complex version of $\mathscr{L}_{2}$ to be

$$
\begin{aligned}
& N_{\mu}=\left(\begin{array}{cc}
\bar{n}_{\mu} & Q_{\mu}^{b} \\
-\frac{1}{2} Q_{\mu}^{a} & n_{\mu}^{a b}
\end{array}\right), \\
& M=\left(\begin{array}{cc}
2 X_{g}+P^{-1} X_{f}+\bar{\tau} & -2 f C^{b a c} Q^{a} V^{c} \\
-g_{\gamma \lambda} \partial_{\mu} Q^{a \rho \sigma \mu \lambda} & Z^{a b}+\tau^{a b}
\end{array}\right)
\end{aligned}
$$

for the $(h, A)$ part, and

$$
\begin{aligned}
& N_{\mu}=\left(\begin{array}{cc}
\bar{m}_{\mu \alpha}{ }^{\beta} & 0 \\
-\frac{1}{2} F^{a}{ }_{\mu}{ }^{\beta} & m_{\mu}{ }^{a b}
\end{array}\right) \\
& M=\left(\begin{array}{cc}
-R_{\alpha}{ }^{\beta}+\bar{u}_{\alpha}{ }^{\beta} & 0 \\
-D^{\lambda} F_{\lambda}^{a \beta} & u^{a b}
\end{array}\right)
\end{aligned}
$$

for the $(\psi, \chi)$ part, respectively. Here

$$
\begin{aligned}
& \bar{n}_{\alpha \beta}^{\mu}{ }^{\rho \sigma}=-2 g^{\mu \lambda} \Gamma_{\lambda \alpha}^{\rho} g_{\beta}{ }^{\sigma}, \\
& \bar{\tau}_{\alpha \beta}{ }^{\rho \sigma}=\left(\bar{d}_{\mu} \bar{n}^{\mu}+\bar{n}_{\mu} \bar{n}^{\mu}\right)_{\alpha \beta}{ }^{\rho \sigma}, \\
& n_{\alpha}^{\mu a b \beta}=-g^{\mu \nu} \Gamma_{\nu \alpha}^{\beta} \delta^{a b}-f C^{e a b} V_{e}^{\mu} g_{\alpha}^{\beta}, \\
& \tau_{\alpha}^{a b \beta}=\left(\bar{d}_{\mu} n^{\mu}+n_{\mu} n^{\mu}\right)^{a b}{ }_{\alpha}, \\
& \bar{m}^{\mu}{ }_{\alpha}^{\beta}=-g^{\mu \lambda} \Gamma_{\lambda \alpha}^{\beta}, \\
& \bar{u}_{\alpha}{ }^{\beta}=\left(\bar{d}_{\mu} \bar{m}^{\mu}+\bar{m}_{\mu l} \bar{m}^{\mu}\right)_{\alpha}{ }^{\beta}, \\
& m_{\mu}{ }^{a b}=-f C^{e a b} V_{\mu}^{e}, \\
& u^{a b}=\left(\bar{d}_{\mu} m^{\mu}+m_{\mu} m^{\mu}\right)^{a b},
\end{aligned}
$$

where $\bar{d}_{\mu}$ acts only on the index $\mu$.

The counter Lagrangian $\Delta \mathscr{L}$, which eliminates all the one-loop divergences in the dimensionalregularization framework, is given by the lemma ${ }^{2}$ to be

$\Delta \mathscr{L}=\frac{1}{\epsilon}(-g)^{1 / 2} \operatorname{Tr}\left[\frac{1}{12} Y_{\mu \nu} Y^{\mu \nu}+\frac{1}{2} X^{2}+\frac{1}{60}\left(R_{\mu \nu} R^{\mu \nu}-\frac{1}{3} R^{2}\right)\right]$

in terms of the matrices

$$
\begin{aligned}
& Y_{\mu \nu} \equiv \partial_{\mu} N_{\nu}-\partial_{\nu} N_{\mu}+N_{\mu} N_{\nu}-N_{\nu} N_{\mu}, \\
& X \equiv M-D_{\mu} N^{\mu}-N_{\mu} N^{\mu}-\frac{1}{6} R I .
\end{aligned}
$$

The unit matrix $I$ is in the product space of world and isospin indices. 
Before giving the explicit result, we can write down all possible types of counter terms allowed by gauge invariance and dimensional considerations. The external $V$ field has canonical dimension one, while $g_{\mu \nu}$ is dimensionless and $\kappa \sim L^{+1}$. We must therefore find all coordinate and gauge invariant terms of dimension $\mathrm{L}^{-4}$ constructed from the metric, curvature, field strength, and covariant derivatives, and involving $\kappa$ in powers $\kappa^{0}$ to $\kappa^{4}$ (since the $P, X, Q, Z$ involve $\kappa^{0}, \kappa^{1}, \kappa^{2}$ ). Likewise, $f$ can only enter as $f^{0}, f^{1}, f^{2}$. A number of apparently different possibilities can be related; for example,

$$
\begin{aligned}
& R_{\mu \nu \alpha \beta} R^{\mu \alpha \nu \beta}=\frac{1}{2} R_{\mu \nu \alpha \beta} R^{\mu \nu \alpha \beta}, \\
& R^{\mu \nu \alpha \beta} F_{\mu \alpha}^{a} F_{\nu \beta}^{a}=\frac{1}{2} R^{\mu \nu \alpha \beta} F_{\mu \nu}^{a} F_{\alpha \beta}^{a}, \\
& \begin{aligned}
\left(D_{\alpha} F_{\beta \gamma}^{a}\right)\left(D^{\beta} F^{a, \alpha \gamma}\right) \sim & \left(D^{\alpha} F_{\alpha \beta}^{a}\right)^{2}+R^{\alpha \beta \rho \gamma} F_{\alpha \beta}^{a} F_{\rho \gamma}^{a} \\
& +R_{\alpha \beta} F_{2}^{\alpha \beta}+C^{a b c} F_{\mu}^{a \nu} F_{\nu}^{b \beta} F_{\beta}^{c \mu},
\end{aligned}
\end{aligned}
$$

where $\sim$ means that the relation holds under the $\int(-g)^{1 / 2} d^{4} x$ integration. There is also an identity relating quadratic curvatures as follows:

$$
R_{\mu \nu \alpha \beta}^{2} \sim 4 R_{\mu \nu}^{2}-R^{2} .
$$

The most general counterterm of dimension four has the form

$$
\begin{aligned}
\Delta \mathcal{L}=\frac{1}{\epsilon}(-g)^{1 / 2}[ & a_{1} R_{\mu \nu}{ }^{2}+a_{2} R^{2}+a_{3} \kappa^{2} R_{\mu \nu} T^{\mu \nu}+a_{4} \kappa^{2} R F^{2}+a_{5} \kappa^{2} R^{\mu \nu \rho \sigma} F_{\mu \nu}^{a} F_{\rho \sigma}^{a}+a_{6} \kappa^{4} T_{\mu \nu}{ }^{2}+a_{7} \kappa^{4}\left(F^{a} F^{b} F^{a} F^{b}\right) \\
& \left.+a_{8} \kappa^{4}\left(F^{a} F^{a}\right)^{2}+a_{9} \kappa^{4}\left(F^{a} F^{b}\right)^{2}+a_{10} \kappa^{2}\left(D^{\mu} F_{\mu \nu}^{a}\right)^{2}+a_{11} \kappa^{2}\left(D_{\alpha} F_{\beta \nu}^{a}\right)^{2}+a_{12} \kappa^{2} f C^{a b c}\left(F^{a} F^{b} F^{c}\right)+a_{13} f^{2} F^{2}\right],
\end{aligned}
$$

where $\epsilon=8 \pi^{2}(n-4)$ and the symbol ( ) stands for the trace over Lorentz indices. The $\kappa, f$ dependence of the $a_{i}$ can be determined by referring to the basic building blocks; as defined, the $a_{i}$ are $\kappa-, f$-independent, but can involve the group dimension $r$ (the above dependence on coupling constants could also be inferred by redefining $V_{\mu}^{\prime a}=\kappa^{-1} V_{\mu}^{a}$, and noting that $f$ only enters in the dimensional combination $\left.f / \kappa\right)$.

Returning to our calculation, we first determine the nonghost matrices of Eq. (2.14),

$$
Y_{\mu \nu}=\left(\begin{array}{cc}
S_{\mu \nu}-\frac{1}{2} Q_{\mu} Q_{\nu}+\frac{1}{2} Q_{\nu} Q_{\mu} & \left(D_{\mu} Q_{\nu}-D_{\nu} Q_{\mu}\right)^{b} \\
-\frac{1}{2}\left(D_{\mu} Q_{\nu}-D_{\nu} Q_{\mu}\right)^{a} & -R_{\mu \nu} \delta^{a b}-f C^{e a b} F_{\mu \nu}^{e}-\frac{1}{2} Q_{\mu}^{a} Q_{\nu}^{b}+\frac{1}{2} Q_{\nu}^{a} Q_{\mu}^{b}
\end{array}\right)
$$

with $S_{\mu \nu}^{\alpha \beta, \rho \sigma}=-2 g^{\alpha \rho} R_{\mu \nu}^{\sigma \beta}$, symmetrized on each pair $(\alpha \beta),(\rho \sigma)$ and under interchange of these pairs. Next,

$$
X=\left(\begin{array}{cc}
2 X_{g}+P^{-1} X_{f}+\frac{1}{2} Q_{\mu} Q^{\mu}-\frac{1}{6} R & -D_{\mu} Q^{\mu} \\
-\frac{1}{2} D_{\mu} Q^{\mu} & Z+\frac{1}{2} Q_{\mu} Q^{\mu}-\frac{1}{6} R
\end{array}\right),
$$

where we have used $P^{-1} Q=2 Q, P^{-1} X_{g}=2 X_{g}$.

The traces of the diagonal and off-diagonal parts in $Y^{2}$ are given by

$$
\begin{aligned}
& {\left[S_{\mu \nu}-\frac{1}{2} Q_{\mu} Q_{\nu}+\frac{1}{2} Q_{\nu} Q_{\mu}\right]^{2}=-6 R_{\alpha \beta \rho \sigma}{ }^{2}-\kappa^{2} R F^{2}+\kappa^{4}\left[3 F^{4}-\frac{3}{4}\left(F^{2}\right)^{2}-\frac{3}{2}\left(F^{a} F^{b}\right)^{2}\right],} \\
& \begin{aligned}
{\left[-R_{\mu \nu} \delta^{a b}-f C^{e a b} F_{\mu \nu}^{e}-\frac{1}{2} Q_{\mu}^{a} Q_{\nu}^{b}+\frac{1}{2} Q_{\nu}^{a} Q_{\mu}^{b}\right]^{2}=} & -\gamma R_{\alpha \beta \rho \sigma}{ }^{2}-2 \kappa^{2} R_{\alpha \beta} F_{2}^{\alpha \beta}-4 f^{2} c_{2} F^{2} \\
& +\kappa^{4}\left[\frac{25}{4} F^{4}-\frac{3}{4}\left(F^{a} F^{b} F^{a} F^{b}\right)-\frac{7}{4}\left(F^{2}\right)^{2}-\frac{3}{2}\left(F^{a} F^{b}\right)^{2}\right],
\end{aligned} \\
& -\left(D_{\mu} Q_{\nu}-D_{\nu} Q_{\mu}\right)^{2}=\kappa^{2}\left[-\frac{9}{2}\left(D_{\mu} F_{\alpha \beta}^{a}\right)^{2}+\left(D_{\mu} F_{a}^{\mu \nu}\right)^{2}\right],
\end{aligned}
$$

with $F^{4} \equiv\left(F^{a} F^{a} F^{b} F^{b}\right), F_{2}^{\alpha \beta} \equiv F_{a}^{\alpha \mu} F_{a \mu}^{\beta}$.

Similarly, the $X^{2}$ terms are given by

$$
\begin{aligned}
& {\left[2 X_{g}+P^{-1} X_{f}+\frac{1}{2} Q^{\mu} Q_{\mu}-\frac{1}{6} R\right]^{2}=6 R_{\mu \nu}{ }^{2} }+\frac{5}{18} R^{2}+\kappa^{4}\left[\frac{1}{2} F^{4}-\frac{1}{8}\left(F^{2}\right)^{2}\right], \\
& \begin{aligned}
{\left[R_{\gamma \delta}+2 f C^{a b e} F_{\gamma \delta}^{e}+\frac{1}{2} Q^{a \mu} Q_{\mu}^{b}-\frac{1}{6} R\right]^{2}=} & r R_{\mu \nu}^{2}-\frac{2}{9} r R^{2}+\kappa^{2} R^{\mu \nu} F_{\mu \nu}^{2}+4 f^{2} C_{2} F^{2} \\
& +\kappa^{4}\left[\frac{1}{8} F^{4}+\frac{1}{8}\left(F^{a} F^{b} F^{a} F^{b}\right)+\frac{1}{2}\left(F^{a} F^{b}\right)^{2}\right]
\end{aligned} \\
& {\left[D_{\mu} Q_{a}^{\mu}\right]^{2}=\kappa^{2}\left[\frac{3}{4}\left(D_{\mu} F_{\lambda \nu}^{a}\right)^{2}+\frac{1}{2}\left(D_{\mu} F_{a}^{\mu \nu}\right)^{2}\right], }
\end{aligned}
$$

where $r C_{2} \equiv C^{a b c} C_{a b c}$. Adding up the above contributions, we obtain for the nonghost counterterm (after dividing by 2 because of the complex fields) 


$$
\Delta \mathscr{L}_{\mathrm{ng}}=\frac{1}{\epsilon}(-g)^{1 / 2}\left\{\left[\frac{7}{10}+\frac{7}{60}(r-1)\right] R_{\mu \nu}^{2}+\left(\frac{4}{15}-\frac{r-1}{40}\right) R^{2}+\frac{1}{6} \kappa^{2} R_{\mu \nu} T^{\mu \nu}+\frac{1}{6} \kappa^{2}\left(D_{\mu} F_{a}^{\mu \nu}\right)^{2}+\frac{13}{24} \kappa^{4} T_{\mu \nu}^{2}+f^{2} C_{2} \frac{5}{6} F^{2}\right\} .
$$

The ghost matrices of Eq. (2.14) have the form

$$
\begin{aligned}
& Y_{\mu \nu}=\left(\begin{array}{cc}
R^{\beta}{ }_{\alpha \mu \nu} & 0 \\
-\frac{1}{2}\left(D_{\mu} F_{\nu}^{a \beta}-D_{\nu} F_{\mu}^{a \beta}\right) & -f C^{e a b} F_{\mu \nu}^{e}
\end{array}\right), \\
& X=\left(\begin{array}{cc}
-R_{\alpha}^{\beta}-\frac{1}{6} R & 0 \\
-\frac{1}{2} D^{\lambda} F_{\lambda}^{a \beta} & -\frac{1}{6} R
\end{array}\right) .
\end{aligned}
$$

Hence

$$
\begin{aligned}
& \operatorname{Tr} Y_{\mu \nu}^{2}=-R_{\alpha \beta \mu \nu}{ }^{2}-f^{2} C_{2} F^{2}, \\
& \operatorname{Tr} X^{2}=\left(\frac{1}{4}+\frac{r-1}{60}\right) R_{\mu \nu}^{2}+\left(\frac{7}{24}+\frac{r-1}{120}\right) R^{2} .
\end{aligned}
$$

The ghost counterterm is then, from Eq. (2.13) and adding a minus sign for the ghost loop,

$$
\begin{aligned}
\Delta \mathcal{L}_{g}=-\frac{1}{\epsilon}(-g)^{1 / 2} & {\left[\left(\frac{1}{4}+\frac{r-1}{60}\right) R_{\mu \nu}{ }^{2}+\left(\frac{7}{24}+\frac{r-1}{120}\right) R^{2}\right.} \\
& \left.-\frac{f^{2} C_{2}}{12} F^{2}\right] .
\end{aligned}
$$

The total one-loop counter Lagrangian is therefore Eq. (2.20) plus Eq. (2.23), namely,

$$
\begin{aligned}
\Delta \mathscr{L}=\frac{1}{\epsilon}(-g)^{1 / 2} & {\left[\left(\frac{9}{20}+\frac{r-1}{10}\right) R_{\mu \nu}{ }^{2}-\left(\frac{1}{40}+\frac{r-1}{30}\right) R^{2}\right.} \\
& +\frac{\kappa^{2}}{6} R_{\mu \nu} T^{\mu \nu}+\frac{\kappa^{2}}{6}\left(D_{\mu} F_{a}^{\mu \nu}\right)^{2} \\
& \left.+\frac{13}{24} \kappa^{4} T_{\mu \nu}{ }^{2}+f^{2} C_{2} \frac{11}{12} F^{2}\right] .
\end{aligned}
$$

Note that most of the possible terms given in (2.15) have "miraculously" canceled out, and indeed, the self-interactions of the gauge multiplet did not generate any new counterterms (other than the $F^{2}$ term) different from those of a set of $r$ Abelian photons. We are only interested in on-mass-shell renormalizability (we are not concerned here with the infrared problem), so it suffices to insert the Einstein-Yang-Mills field equations, obeyed by the background fields, to obtain

$$
\Delta \mathscr{L}=\frac{1}{\epsilon}(-g)^{1 / 2}\left[\left(\frac{137}{60}+\frac{r-1}{10}\right) R_{\mu \nu}^{2}+f^{2} C_{2} \frac{11}{12} F^{2}\right] .
$$

There are two parts to $\Delta \mathcal{L}$; the $R_{\mu \nu}^{2}$ part is just that due to a sum of $r$ noninteracting photons, since $\frac{137}{60}$ is the result for a single Maxwell field, while $\frac{1}{10} R_{\mu \nu}{ }^{2}$ is the contribution of a single photon loop $^{3}$ (including its ghost part) to $\Delta \mathscr{L}$. The second term is, of course, just the usual flat-space Yang-Mills counter term, and can be removed by the usual (infinite) rescaling of $V$ and $f$ in the original action. However, the $R_{\mu \nu}{ }^{2}$ term alone suffices, as in the Maxwell case, to imply that the one-loop divergences cannot be removed by the conventional rescaling.

These results provide the explicit reasons why the parameters $r$ and $f$ could not be used to cancel counter terms. The multiplicity of the fields enters only in the $R_{\mu \nu}{ }^{2}$ term, but the latter can be shown on general grounds (see Appendix of Ref. 3) to be positive-definite, and indeed each Yang-Mills component contributed the same amount additively. Likewise, the fact that the self-coupling constant could only enter in the combination $(f / \kappa)$ and therefore, could only appear in front of an $F^{3}$ term (aside from the $F^{2}$ ), ruled out its usefulness.

\section{YANG-MILLS AND MAXWELL COUNTERTERMS}

In this section we give a qualitative derivation, using only the known Einstein-Maxwell counter terms, gauge invariance, and dimensional consideration of the type of divergence which was found in Sec. 2. Consider the general possible $\Delta \mathfrak{L}$ of Eq. (2.15), which is written down using only gauge invariance and dimensions. The term $a_{13}$ is just the usual flat-space Yang-Mills counterterm and its presence is in any case harmless, since it can be renormalized away by the usual scale changes. The $a_{2}, a_{4}$, and $a_{10}$ terms all vanish on the mass shell. This leaves three remaining classes of dangerous terms: the $\left(a_{1}, a_{3}, a_{6}\right)$ group which becomes proportional to $R_{\mu \nu}{ }^{2}$ using the Einstein equation, the purely non-Abelian terms $\left(a_{7}, a_{9}, a_{12}\right)$ and the possible Abelian terms $\left(a_{5}, a_{8}, a_{11}\right)$. Now the presence of any of these latter six terms would already imply non renormalizability since they are not removable either by rescaling, nor can they be affected by the onmass-shell equations. Whether they are there or not, however, we can also say a great deal about the first group $\left(a_{1}, a_{3}, a_{6}\right)$. Since the general form for $\Delta \mathscr{L}$ holds for every value of $f$ including $f=0$, and we know that none of the $a_{i}$ can depend on $f$ (see end of Sec. 2), but only on $r$, we know that the value $a_{3}$ must be the same as in the Maxwell 
case (just take the $f=0$ limit), while $a_{6}$ will also have the Maxwell value if $a_{7}$ and $a_{9}$ vanish (and in any case the sums of $a_{6}, a_{7}$ with $a_{8}$ and $a_{9}$ must agree with the Maxwell values). The terms $a_{5}, a_{8}, a_{11}$, are identical in form in both Abelian and non-Abelian cases and therefore must vanish here also (they are anyhow independent of the rest, as noted, and intrinsically "bad"). This leaves the $a_{1}$ coefficient which could be (and indeed is) $r$-dependent. Here, however, we may argue on unitarity grounds that this $r$ dependence cannot decrease $a_{1}$ below its Maxwell value, i.e., $a_{1}(r)$ $\geqslant a_{1}(1)$. [In fact, we can show, again using the $f=0$ limit, that $a_{1}(r)$ is equal to value $a_{1}(1)$ plus $(r-1)$ times the (positive) contribution to $R_{\mu \nu}{ }^{2}$ from a pure photon loop.] Now, also $a_{6}(r)$ will either equal $a_{6}(1)$ or the "bad" $a_{7}, a_{9}$ terms will persist. But the field equations $R_{\mu \nu}=-\frac{1}{2} T_{\mu \nu}$ combine $a_{1}, a_{3}, a_{6}$ to be $\left(a_{1}-2 a_{3}+4 a_{6}\right) R_{\mu \nu}{ }^{2}$, and we know that at $r=1$, this combination is positive. Thus, it must be positive here as well, since only $a_{1}$ can change, and it can only increase. The above argument ${ }^{5}$ is thus sufficient to exhibit the existence of new counter terms, though it cannot of course display the surprising numerical cancellation of most of the terms in question, particularly $a_{7}, a_{9}$, and $a_{12}$; only the extensive calculations described in Sec. II could yield their specific values.

\section{CONCLUSIONS}

Our result has been to add non-Abelian vector gauge fields to the list of systems whose interactions with the Einstein field are not one-loop renormalizable in the usual sense(although the unlikely possibility that no further counterterms might be needed at higher loops must still be borne in mind). Other self-interacting systems, perhaps with different gauge properties, might fare better. The recently introduced "supergauge" multiplets ${ }^{6}$ combining half-integral and integral spins provide an example to be investigated.

The form of the one-loop counter terms suggests another line of speculation concerning possible improvement of the ultraviolet problem. Consider first pure gravity, where $\Delta \mathscr{L}$ has the form $\alpha R_{\mu \nu}{ }^{2}$ $+\beta R^{2}$. In computing two-loop divergences, we find that the effect of $\Delta \mathscr{L}$, treated as a perturbation, is then only to make matters worse, and to lead to possible $\kappa^{2} R_{\mu \nu \alpha \beta}{ }^{3}$ and higher-power terms. However, one can adopt the point of view (reviving a speculation by DeWitt and other $\mathrm{s}^{7}$ ) that the correct Lagrangian should be of the form $\mathscr{L}=\left(1 / \kappa^{2}\right) R+\alpha R_{\mu \nu}{ }^{2}+\beta R^{2}$ to begin with, i.e., a combination of Einstein and $\mathrm{Weyl}^{8}$ models. It has one manifest advantage. Since the weak field propagators in this model are of the form $\left(p^{2}\right.$ $\left.+\gamma^{2} \kappa^{2} p^{4}\right)^{-1}$, while the vertices behave at most as $p^{+1}$, the form of all-order-loop contributions will preserve the structure of $\mathcal{L}$, and the theory is therefore renormalizable by power counting alone. From the structure of the propagator, we notice that $\kappa^{-1}$ acts as a natural momentum cutoff. Coupling to matter will retain renormalizability, because the matter-graviton vertices are not altered ${ }^{9}$ (we retain minimal coupling), but only the graviton propagator is improved.

Before taking this model seriously as a physical reasonable theory, a number of very basic questions must be faced, however. The first requirement is of course that the slowly varying weak field results of classical Newtonian theory be reproduced. Roughly speaking, the Poisson equation will become something like $\left(\gamma^{2} \kappa^{2} \nabla^{4}+\nabla^{2}\right) \phi=\kappa^{2} \rho$. While the pure Weyl theory is clearly in trouble [ since $\nabla^{-4} m \delta^{3}(r) \sim m r$ asymptotically], the present combination produces the modified potential $\phi \sim \kappa^{2} M / r[1-\exp (-r / \gamma \kappa)]$, which agrees with the Newtonian one for large $r$. Secondly, one must make sure that an appropriate combination of $R_{\mu \nu}{ }^{2}$ and $R^{2}$ can be found such that the propagators of all components of the metric have the leading $p^{-4}$ behavior, and that no extraneous helicity components are introduced [obvious candidates are the combination $\left(R_{\mu \nu}{ }^{2}-\frac{1}{3} R^{2}\right)$ corresponding to the Weyl tensor squared and $\left.\left(R_{\mu \nu}{ }^{2}-\frac{1}{2} R^{2}\right)\right]$. Third$1 y$, we note the most obvious question at the quantum level of the traditional difficulty associated with higher derivative theories, ${ }^{10}$ namely, whether there will be ghosts. It is fairly clear that pure Weyl theory with pure $p^{-4}$ propagators would suffer from this difficulty. In the present case, one would expect a ghost to appear as a particle with mass $\sim \kappa^{-1}$, in addition to the massless graviton. Finally we note that the present scheme falls outside the standard perturbative coupling constant expansion framework. Although one still expands vertices in powers of $\kappa$ (i.e., in powers of the quantum field $h$ ) the propagator structure has additional nonpolynomial $\kappa$ dependence. 
*Research supported by the National Science Foundation. †Research supported by the U. S. Atomic Energy Commission under Contract No. AT (11-1)3230. Address after September 1974: Institute for Advanced Study, Princeton, N. J. 08540.

$\$$ Research supported by the National Science Foundation and Nederlands Ministerie van Onderwijs en Wetenschappen.

${ }^{1}$ A summary of the results of this paper was given by S. Deser, H.-S. Tsao, and P. van Nieuwenhuizen, Phys. Lett. 50B, 491 (1974).

${ }^{2} \mathrm{G}$. 't $\mathrm{Hooft}$ and M. Veltman, Ann. Inst. Henri Poincaré (to be published); G. 't Hooft, Nucl. Phys. B62, 444 (1973).

${ }^{3}$ S. Deser and P. van Nieuwenhuizen, Phys. Rev. Lett. 32, 245 (1974); Phys. Rev. D 10,401 (1974); 10, 411 (1974).

${ }^{4}$ Renormalizability in the usual sense could still be obtained if only a finite number of counterterms is used to eliminate all divergences. It is still possible that from a certain loop number on no new counterterms are needed.

${ }^{5}$ One can spell out some of the steps of this section in more detail. The $R_{\mu \nu}{ }^{2}$ contribution consists of a pure graviton-loop part (which is of course unaffected by the coupling) and pure particle loops. Each member of the multiplet contributes the same (intrinsically positive) amount as a Maxwell field, because $R_{\mu \nu}^{2}$ divergences can only come from the $\left(\partial_{\mu} A_{\nu}^{a}-\partial_{\nu} A_{\mu}^{a}\right)$ parts of the in- ternal lines (by power counting). The $R_{\mu \nu} T^{\mu \nu}$ coefficient is identical to the Maxwell one: First, there is only one possible way to combine two $F^{a}{ }_{\mu \nu}$, namely, as a scalar product on internal indices; second, it is $f$-independent and must therefore equal its value at $f=0$, where $T^{\mu \nu}$ would be the sum of the individual Maxwell stress tensors. Finally, the coefficient of the usual Yang-Mills counterterm, $f^{2} F^{2}$, is the same as in flat space, since it is $K$-independent by dimensions. ${ }^{6} \mathrm{~J}$. Wess and B. Zumino, Nucl. Phys. B70, 39 (1974); A. Salam and J. Strathdee, ibid. B76, 477 (1974).

${ }^{7}$ B. S. DeWitt, in Relativity, Groups and Topology, edited by C. DeWitt and B. S. DeWitt (Gordon and Breach, London, 1964); B. S. DeWitt and R. Utiyama, J. Math. Phys. 3, 608 (1962); A. D. Sakarov, Dokl. Akad. Nauk SSSR 177, 70 (1968) [Sov. Phys._Dokl. 12, 1040 (1968)]; S. Weinberg (private communication) also advocates investigating models of this type.

${ }^{8} \mathrm{H}$. Weyl, Space, Time, Matter (Dover, New York, 1950). A cosmological term will also have to be present in general, since counterterms of this form could arise. This would mean that in the unrenormalized action asymptotic flatness is absent.

${ }^{9}$ In the case of a scalar field, however, the famous "nonminimal" term $R \phi^{2}$ should be included, since its coupling constant is also dimensionless. Note also that such divergent terms as $\psi^{8}$ in the Dirac-Einstein case (Ref. 3) will no longer be present.

${ }^{10}$ A. Pais and G. Uhlenbeck, Phys. Rev. 79, 145 (1950). 